\title{
On a possibility of parametric amplifier in the stratosphere-mesosphere suggested by active MASSA experiments with the AUREOL-3 satellite
}

\author{
Yu. Galperin ${ }^{1}$ and M. Hayakawa ${ }^{2}$ \\ ${ }^{1}$ Space Research Institute, 117810, Moscow, Russia \\ ${ }^{2}$ University of Electro-Communications, Chofu-shi, Tokyo, Japan \\ (Received October 30, 1997; Revised June 3, 1998; Accepted August 28, 1998)
}

\begin{abstract}
A short review is given of the contemporary concepts and controversies concerning the electromagnetic effects observed in space which are considered to be the results of energy transport from surface and/or lower atmosphere. A concept of a parametric amplifier in the stratosphere-mesosphere was recently suggested by the authors (1996) from the reanalysis of the experimental results from the AUREOL-3 satellite during active experiments MASSA with ground chemical explosions. Electric discharges in the stratosphere-mesosphere medium were suggested as a mechanism for this parametric amplifier. Such discharges, or localized electric current bursts in the Alfven wave frequency scale $\left(\sim 0.1-1 \mathrm{~s}^{-1}\right)$, could also play a role as a mechanism of effective energy transport from the surface to space layers. Among the possible EQ precursor effects, some can be related to local atmospheric electricity variations such as emanations of gas and/or aerosols, conductivity changes in the lower atmosphere, or non-linear combinations of acoustic waves with a meteor ionization channel. Thus a multidisciplinary search of EQ precursors, especially with regard to atmospheric electricity variations, lightning-type, or slower discharges related to meteor ionization, seems to be among the ways to deepen the understanding of the complicated relations between different layers of atmosphere and magnetosphere.
\end{abstract}

\section{Introduction}

A paradoxical situation has developed in the search, and studies, of the electromagnetic (EM) and ionospheric precursors of strong earthquakes (EQ). From one side some weak but measurable EM signals were noted on several satellites when looking at the times of the satellite's passage near future epicentral zones by Gokhberg et al. $(1982,1983)$ and Larkina et al. (1983). The analysis of a large body of ELF/VLF data from the INTERKOSMOS-19 by Larkina $e t$ al. (1989), INTERKOSMOS-24 by Molchanov et al. (1993), and AUREOL-3 by Parrot (1994), have indicated some statistically significant positive correlations with the EQ. The respective signals were measured by narrow (10\%) filters, and are comparable in intensity with other natural ELF/VLF signals (see, reviews by Molchanov (1993), and by Hayakawa (1997)). But, no EQ-related effects were seen in the ISIS wide band data (and respectively, at lower sensitivity) according to Roger et al. (1996). A search of such effects in the DE-2 data by comparing the ELF/VLF intensities on the closest to epicenter and a neighbor pass by Henderson et al. (1993) did not reveal significant differences and thus was interpreted as a negative result. However, according to the above mentioned results with positive correlations, the longitudinal extent of the zone with enhanced ELF/VLF intensities includes several passes. Thus methodical differences between the instruments and antennas on the satellites, data selection and treatment procedures (different wave components, sensitivity, frequency bandwidth, etc.) could be im-

Copy right (C) The Society of Geomagnetism and Earth, Planetary and Space Sciences (SGEPSS); The Seismological Society of Japan; The Volcanological Society of Japan; The Geodetic Society of Japan; The Japanese Society for Planetary Sciences. portant in this controversy, but were not checked properly. Still it can be stated that up till now there are no confirmations/identifications of the ELF/VLF signal signatures measured by other satellites which are definitely related to EQ electromagnetic precursors.

Energetic particle precipitation intensities were also found to have statistically significant increases in such regions (Galper et al., 1989; Galperin et al., 1992; Ginzburg et al., 1994). It could be supposed that some unidentified waveparticle interactions with enhanced ELF/VLF noises were responsible for the scattering and precipitation of high energy trapped electrons on these low L-shells. Besides that, EQ-precursor-related effects were claimed to be observed in ionospheric variations (Fatkullin et al., 1989) and in airglow variations (Fishkova et al., 1985). These latter, non-EM observations apparently were not challenged by other measurements.

The obvious conclusion from these results seems to be that the respective EM signals are either too weak, or short-lived (or both) to be reliably detected from a fast moving nearEarth satellite with the sensitivity, orbits and data treatment procedures used (see, Johnston, 1997). It does not exclude possible existence of such signals by principle, but indicates the inadequacy of the measurement techniques applied to delineate them with a reliable accuracy and repeatability. And evidently, the main problems remain on the exact physical nature of the effects registered, i.e. on the reality of their correspondence to the incipient EQ, and, if positive, on the way of energy transport from the epicentral zone to space. These problems are under investigation by several groups, and undoubtedly their results will contribute to the understanding 
of physical processes in the atmosphere, low-latitude ionosphere and magnetosphere (Molchanov et al., 1995).

From another side, a purely agnostic paper on the subject of the EQ prognosis has recently appeared in "Science" by Geller et al. (1997), with a bold title "Earthquakes cannot be predicted". The authors' view expressed in the title is based on their definition of the "earthquake prediction" as giving the exact time, place and intensity of the incipient EQ. While to fit such stringent requirements seems indeed unfeasible nowadays, an analysis and search seem really necessary and feasible for various physical effects which accompany the stress build up, and can propagate in one or another form to the Earth's surface, or to space, and thus could serve as the EQ precursors. Indeed, for a physicist, it would seem rather improbable that a great accumulation of stress energy, lasting sometimes decades and decades for a large EQ, proceeds without any leakage of energy in any form, i.e. constitutes an ideal lossless accumulator of growing energy. It is evident that search of potential precursors must not be limited to one or another direction, but needs to be as widespread and multidisciplinary as possible (see, Rikitake, 1988).

One of examples of new approaches to describe the physics of the preshock phenomena was recently presented by Gufeld et al. (1998). It is based on a quite reasonable supposition of the hydrogen and helium diffusion from internal regions of the fault zone. While the diffusion from deep layers is rather slow, many but not reliable indications of the gas appearance before EQ exist in literature, they include hydrogen, helium, methane, radon (King, 1986). The point made by Gufeld et al. (1998) is that absorption of these gases in crystals composing rocks can impose their restructuring (well known in solid-state physics), which can lead to local changes of mechanical properties of the medium along a fault, and ultimately to the stress failure and shock.

It is also conceivable that due to the induced crystal restructuring processes the average conductivity of the medium along the fault will be changed, so that remote conductivity change diagnostics from the surface will be possible, at least for shallow epicentral zones. It can be noted that the fitting of a model where a body of enhanced conductivity along the fault was supposed by Merzer (1992) allowed to reproduce the wide spectrum and intensity of the ULF noise enhancements registered before the Loma Prieta EQ (Fraser-Smith et al., 1990). Similar ULF noise enhancements were found also for the Spitak, 1991 EQ (Molchanov et al., 1992) and Guam, 1993 EQ (Hayakawa et al., 1996), but were not found in some other EQs (Fraser-Smith et al., 1994).

A line of research which could be related to the pre-EQ gas emanations is to study a possible role of an enhancement of atmospheric aerosols, injections of which are sometimes believed to be also related to changes in the environment above the incipient EQ (Pulinets, 1998). While the measurements are scarce and not quite convincing, local changes in atmospheric electricity due to aerosols are conceivable. Also, a transport of charged aerosols upward in the atmosphere can be accelerated by atmospheric electric field, at least in fair weather conditions, so they can contribute to the propagation of a disturbance from the ground level to stratosphere-mesosphere.

An interesting prediction method was suggested by Gorny et al. (1988). It includes geographical identifications and local development of the temperature (or intensity) changes within the $15 \mu$ band of $\mathrm{CO}_{2}$ as observed remotely from weather satellites. This approach is currently used for a regular EQ prediction in the northern China and the reported results are exciting according to Qiang Zuji (1991). In fact, the observed intensity changes could be also related to the gas emanations from the fault zones, not necessarily to the surface temperature, but this point could be checked experimentally.

A very promising approach, a modified form of the VLF phase difference method proposed earlier by Gokhberg et al. (1989), is now being developed by Hayakawa, Molchanov and their colleagues (Molchanov et al., 1998). It consists of measuring the deviations of the diurnal VLF phase curve from the predictable form during morning and evening twilight at the $D$-region. It allows for remote observations above an extended area when several transmitters and receivers are used, and has already demonstrated very positive results.

Another potentially very important approach suggested some time ago by Hayakawa et al. (1993), is low-frequency direction-finding experiments to locate the sources of electromagnetic emissions which could be related to incipient earthquakes. Recently a realisation of these ideas has brought the first very promising results (see, Fujinawa et al., 1997). These experiment demonstrated the appearance and motions of sources of impulsive VLF emissions above the incipient epicenters and hundreds of $\mathrm{km}$ around. While the precision of the altitude determinations in these first experiments is to be improved, nevertheless they indicate that the VLF source locations can be in the troposphere and/or mesosphere above a broad region near the incipient epicenter for a large EQ. Evidently, this is also a promising direction of experimental research in the subject.

Thus it seems for us that if unreasonably stringent requirements are not put forward, and the analysis is directed to physical and chemical processes potentially related to the development of internal stresses which ultimately may lead to $\mathrm{EQ}$, a fruitful multidirectional area of research for potential EQ precursors exists and is expanding. As the physical conditions and characteristics of particular "precursors" will be identified and understood on physical grounds, their practical validity and usefulness for a prediction, as well as their possible precision and error probability, will be evaluated. But the view expressed by Geller et al. (1997) on the principal non-solubility, or non-feasibility of the problem, seems to us as rather "extreme" and ungrounded.

\section{EQ Precursors Observed in Space: Reality or Myth?}

Among several EQ precursor effects claimed to be observed in space there are ELF/VLF noises, energetic particle precipitations, large-scale ionospheric density variations and airglow variations. To be real, a way (or ways) for the energy transport is to be found from the incipient EQ epicenter (underground) to ionospheric altitudes. This energy transport must be such that it is not easily distinguishable from the surface, otherwise it would be found long ago and used for prediction purposes.

An evaluation of the energy required for the ionospheric 
and airglow modifications observed and claimed to be EQrelated was made in Galperin et al. (1992). It was shown that the amplitude of surface oscillations, needed to excite a suitable acoustic energy flux, sufficient to excite variations in the thermosphere leading to disturbances in ionosphere and airglow, must have amplitudes of order of $1 \mathrm{~mm}$ - a value orders of magnitude higher than the sensitivity threshold of contemporary measurement techniques. Thus the purely acoustic way of the energy transport from the surface to ionospheric altitudes sufficient to produce all the claimed precursory effects, is thus rejected.

For the electromagnetic way of energy transport directly from the epicenter zone to the ionosphere, extensive calculations were made in Molchanov et al. (1995). It was shown that the maximal feasible intensities of electromagnetic signals excited within the epicenter zone are at least an order of magnitude lower than were observed from satellites. Thus this way of the energy transport to ionosphere/magnetosphere is also rejected.

What other ways are possible? Or, if no other ways are found, are the space effects claimed to be EQ-related are in fact just random coincidences, i.e. is it a myth in connection to the EQ-precursor research?

\section{Active Experiments-A "Natural" Modeling of Energy Transport from Surface to Space}

A surface disturbance in the form of an acoustic shock produced by a chemical explosion, could serve to make a "natural" model to study a response of the ionosphere/magnetosphere system. To this end, a series of active multidisciplinary experiments was performed in 1981-1983 under the name MASSA (Gokhberg, 1983). The coordinated measurements in space were performed with the AUREOL-3 satellite (see, Galperin et al., 1985a,b; Galperin and Hayakawa, 1996 and references therein).

In this series of active experiments several surface explosions were used in the range of a fraction of a kiloton to several kilotons TNT, which were coordinated with the passes of the AUREOL-3 satellite in the near vicinity of the Explosion Flux Tube (EFT). The EFT was defined as the magnetic flux tube with its base centered on the point at $100 \mathrm{~km}$ altitude vertically above the explosion point on the ground. The target parameter (the minimal distance between the satellite trajectory and the EFT axis) ranged from less than $50 \mathrm{~km}$ to more than $200 \mathrm{~km}$, and the time of the satellite closest approach to the EFT axis ranged from $\sim 220 \mathrm{~s}$ to $20 \mathrm{~min}$, and in one case even by nearly 4 hours due to a programming error.

All the experiments according to Galperin et al. (1985a,b) have consistently shown rather strong effects within the EFT in the form of wideband noises extended from ELF to VLF range in the altitude range from the perigee to apogee of the AUREOL-3 (400-2000 km). The diameter of the noise zone around the EFT expanded with time with about the sound speed in the thermosphere $(\sim 600 \mathrm{~m} / \mathrm{s})$ up to at least $1000 \mathrm{~km}$ distance. These enhanced noises were detectable even nearly 4 hours after one of the explosions. The general characteristics of these noises were rather similar to those claimed to be EQ-related. For the two experiments where the target parameter was $\leq 50 \mathrm{~km}$, another feature-an intense Alfven wave with amplitude $119 \mathrm{nT}$ (MASSA-1) and 50 nT
(MASSA-3) was observed at $L \approx 1.3$ (while the EFT had occurred at $L \approx 1.5$ ).

In short, the reanalysis of the results of these active experiments made by Galperin and Hayakawa (1996) have led to the following unexpected conclusions:

1) The effects observed in the upper ionosphere have started rather early, when the acoustic shock wave was not higher than $70 \mathrm{~km}$. This means that the main effects were not related to the dynamo action of an acoustic wave, as below $70 \mathrm{~km}$ both ions and electrons are not magnetized.

2) The energy flux of the Alfven wave (registered fully, i.e. all 6 components of the Poynting vector, only in MASSA-1, and with only 5 components in the MASSA-3) was at least two orders of magnitude higher than that which could be expected from an analysis of the acoustic wave entrance into the lower ionosphere according to calculations in Danilov and Dovzhenko (1987) and Surkov (1992).

3) All the measured characteristics of the Alfven wave and its shock front as registered in MASSA-1, could be reasonably accounted for by a contemporary theoretical analysis, except its excitation mechanism leading to the observed exceptionally high amplitude. Also, these noises can persist in the mid-latitude upper ionosphere, in the form of weak electrostatic noises, for a very long time, at least for several hours. It may be added that recent analysis of these data by Pokhotelov et al. (1994) indicates that ion-cyclotron instability can also play a role in the Alfven shock front in addition to (or instead of) the ion-acoustic one considered in earlier papers on the subject.

It was concluded by Galperin and Hayakawa (1996) from these reanalyses of the MASSA experiments with the AUREOL-3 satellite that there exists some very efficient mechanism of the energy transport from surface to space, a kind of a parametric amplifier in the atmosphere. This mechanism can transport, and probably amplify, the disturbance energy in such a way that it excites the ELF/VLF noises in the upper ionosphere/magnetosphere along the EFT. The noise characteristics observed are rather similar to those observed earlier in space on this and other satellites which were claimed to be EQ-precursor related.

Thus the MASSA experiments, while originally directed to check the validity of the acoustic dynamo-action approach in the EQ-precursor problem, in fact have revealed the existence of another, still unidentified but powerful mechanism of the energy transport from the surface to space. This mechanism by its energetics is potentially capable to excite the observed space effects of the types that were claimed to be EQ-precursor related.

\section{Speculations about the Role of Atmospheric Electricity in a Parametric Amplifier in the Stratosphere-Mesosphere}

It was further suggested by Galperin and Hayakawa (1996) that the mechanism of the parametric amplifier could be some form of a stimulated electric discharge similar to "Red Sprites", or "Blue Jets" sometimes observed during strong lightnings. These troposphere-stratosphere-mesospherelower ionosphere discharges are widely discussed now in the literature (see Sentman and Wescott (1993) and Winckler (1995), for reviews of optical observations, and Dowden 
(1996), Dowden et al. (1996), and Inan et al. (1996) for a discussion of the related VLF perturbations and their interpretation).

Some new approaches to theoretically describe the atmospheric chemistry and ionization changes in the presence of electric discharges and non-linear acoustic waves were introduced by Martysh and Sidorenko (1997) (see also, Devyaterikov et al., 1984). To our opinion, these calculations open a possibility of a non-linear evolution of an enhancement of electron density at the ascending front of an acoustic wave in real variable conditions in the mesosphere which could lead to observable consequences, in particular, in local atmospheric electricity conditions.

Atmospheric electricity changes are often claimed to be among the precursor phenomena (see, for example, Finkelstein and Powell, 1970; Finkelstein et al., 1973; Pierce, 1976). It is known that effects of an electric discharge from electrified thundercloud, or surface, to the ionosphere and magnetosphere can be very significant (see, Farrell et al., 1994; Rowland et al., 1995; Sukhorukov et al., 1996; Roussel-Dupre et al., 1994). They can include so called Trimpi effects with generation of powerful whistler waves and resulting enhancement of particle precipitation. Energetically they are capable to induce air pressure impulses with resulting neutral air motions and airglow variations, ionospheric disturbances and electromagnetic emissions, i.e. all the effects claimed to be EQ-precursors in space. However, to our knowledge, up till now no direct physical links between atmospheric electricity variations and EQ precursor effects were formulated in a model suitable for an experimental test.

Recently it was directly observed from space by Burke et al. (1992), that a strong lightning discharge below a satellite excited a local field-aligned current, and thus an Alfven wave. Thus an effectivity of a lightning discharge to excite Alfven waves in the magnetosphere originally predicted by Greifinger and Greifinger (1976) was directly demonstrated. This observation corroborates with the conclusions in Galperin and Hayakawa (1996) on the possible way of the effective energy transport from lower atmosphere to ionosphere/magnetosphere. To be real, a mechanism needs to be found to produce a local increase of the atmospheric electric field up to the breakdown values (i.e. by orders of magnitude). Evidently, these considerations remain only qualitative at this stage, but could be of interest in a search of possible EQ-precursor-related processes.

At the same time, the first results by Fujinawa et al. (1997) on the observations of impulsive VLF bursts above an incipient EQ, if confirmed by further measurements, give some support to the main suppositions in Galperin et al. (1985a) concerning the existence of a parametric amplifier in the atmosphere, its location in the stratosphere-mesosphere, and its possible electric discharge nature. Evidently a further search would be interesting, both experimental and theoretical, for localized bursts of currents in the middle atmosphere which are distinct from that caused by thundercloud discharges.

There is some evidence that a kind of "slow discharges" apparently is possible. If real, they are not easily measurable without special equipment, when arise in the stratospheremesosphere altitudes, and thus may go unnoticed in many areas. A scenario suggested here is a moderately strong ascending acoustic wave front in the mesosphere which is pierced by a meteor ionization channel. This can produce a localized current (a "slow discharge") in the Alfven wave frequency range $\left(\sim 0.1-1 \mathrm{~s}^{-1}\right)$ between the enhanced electron density at the acoustic wave front, and ionosphere leading to an Alfven wave excitation.

Let us evaluate this possibility to an order of magnitude. Consider the ascending acoustic front in the mesosphere, say, at $60 \mathrm{~km}$ altitude after the MASSA-1 explosion. Electron density in the sunlit $D$-region at this altitude amounts to 100 el. $\mathrm{c}^{-3}$, and somewhat increases within the acoustic wave front (Inan et al., 1996). If a meteor pierced the upper mesosphere at this time near the front center, an ionised conductive column is created between the $E$-region ionosphere and the acoustic wave front. If there exists a potential difference between the two, a short-term magnetic variation will arise, say, during $0.3 \mathrm{~s}$ - the time of meteor crossing of atmospheric scale height. At a distance $100 \mathrm{~m}$ from the ionised column axis magnetic variation of $120 \mathrm{nT}$ (observed amplitude in MASSA-1) needs a current of $\sim 60$ A which during 0.3 s carries the charge $Q=18$ Coulombs. Such a quantity of electrons $N \approx Q \cdot 6 \cdot 10^{18} \approx 1 \cdot 10^{20} \mathrm{el}$. is contained in a volume of acoustic front of $1 \mathrm{~km}$ thickness in the circular area of radius $\sim 30 \mathrm{~km}$. The electron conductivity within the acoustic front is enhanced both due to thermal ionisation of cluster ions (Inan et al., 1996) and also due to the increased temperature.

Thus a "slow discharge" to ionosphere capable to excite a kinetic Alfven wave of the observed large amplitude seems feasible, but needs special conditions - a meteor just in time. It may be noted that the day of the MASSA-1 experiment, November 28, 1981, took place not too far from the maximum, on November 30, of the daytime meteor shower Andromedids (presumably originated from the Biela comet), and at morning local time when the relative average velocity of meteors is at a maximum due to the Earth's orbital motion.

It remains to be seen whether this mechanism can play a role in producing the Alfven waves observed in MASSA experiments and/or those claimed to be EQ precursors in the magnetosphere.

A detailed study of these energy transport processes from surface and lower atmosphere to space through the stratosphere-mesosphere regions (still often called "ignorosphere" due to their complexity) seems to be one of the ways to uncover various physical links between different layers of the atmosphere and magnetosphere.

\section{References}

Burke, W. J., T. L. Aggson, N. C. Maynard, W. R. Hoegy, R. A. Hoffman, R. M. Candy, C. Liebrecht, and E. Rodgers, Effects of a lightning discharge detected by the DE 2 satellite over hurricane Debbie, J. Geophys. Res., 97, 6359-6367, 1992.

Danilov, A. V. and V. A. Dovzhenko, On excitation of electromagnetic fields during acoustic impulse entry in the ionosphere, Geomagn. Aeron., 27, 772-777, 1987 (in Russian).

Devyaterikov, I. A., E. A. Ivanov, S. I. Kozlov, and V. P. Kudryavtsev, Effects of acoustic waves on the behavior of charged particles in the low ionosphere, Cosmic Res., 22, 197-200, 1984 (English translation).

Dowden, P. E., Comment on "VLF signatures of ionospheric disturbances associated with sprites" by Inan et al., Geophys. Res. Lett., 23, 34213422, 1996

Dowden, R. L., J. B. Brundell, W. A. Lyons, and T. Nelson, Detection and 
location of red sprites by VLF scattering of subionospheric transmissions, Geophys. Res. Lett., 23, 1737-1740, 1996.

Farrell, W. M., T. L. Aggson, E. B. Rodgers, and W. B. Hanson, Observation of ionospheric electric fields above atmospheric weather systems, $J$. Geophys. Res., 99, A10, 19475-19483, 1994.

Fatkullin, M. N., T. I. Zelenova, and A. D. Legenka, On the ionospheric effects of asthenospheric earthquake, Phys. Earth Planet. Inter., 57, 82 85, 1989.

Finkelstein, D. and J. R. Powell, Earthquake lightning, Nature, 228, 992, 1970.

Finkelstein, D., R. D. Hill, and J. R. Powell, The piezoelectric theory of earthquake lightning, J. Geophys. Res., 78, 993, 1973.

Fishkova, L. M., M. B. Gokhberg, and V. A. Pilipenko, Relationship between night airglow and seismic activity, Ann. Geophys., 3, 689-694, 1985.

Fraser-Smith, A. C., A. Bernardi, P. R. McGill, M. E. Ladd, R. A. Helliwell, and O. G. Villard, Jr., Low-frequency magnetic field measurements near the epicenter of the Ms 7.1 Loma Prieta earthquake, Geophys. Res. Lett., 17, 1465-1468, 1990.

Fraser-Smith, A. C., P. R. McGill, R. A. Helliwell, and O. G. Villard, Jr., Ultra-low frequency magnetic field measurements in southern California during the Northridge earthquake on 17 January, 1994, Geophys. Res. Lett., 21, 2195-2198, 1994.

Fujinawa, Y. K., K. Takahashi, T. Matsumoto, and N. Kawakami, Experiments to locate sources of earthquake-related VLF electromagnetic signals, Proc. Japan Acad., 73 B, No. 3, 33-38, 1997.

Galper, A. M., V. V. Dmitrenko, N. V. Nikitina, et al., Relation of highenergy charged particles in the radiation belt with the Earth's seismicity, Cosmic Res., 27, 789-792, 1989 (in Russian).

Galperin, Yu. I. and M. Hayakawa, On the magnetospheric effects of experimental ground explosions observed from AUREOL-3, J. Geomag. Geoelectr., 48, 1241-1263, 1996.

Galperin, Yu. I., V. A. Gladyshev, N. V. Jorjio, R. A. Kovrazhkin, Yu. V. Lissakov, V. D. Maslov, L. M. Nikolaenko, R. Z. Sagdeev, O. A. Molchanov, M. M. Mogilevsky, L. S. Alperovich, M. B. Gokhberg, E. A. Ivanov, O. A. Pokhotelov, H. Reme, J.-M. Bosqued, C. Beghin, and J. J. Berthelier, VLF and ELF effects in the upper ionosphere caused by large scale acoustic waves in the lower ionosphere observed from AUREOL-3 satellite, in Results of the ARCAD 3 PROJECT and of the Recent Programmes in the Magnetospheric and Ionospheric Physics, edited by CNES, CEPADUES-EDITIONS., Toulouse, pp. 661-684, 1985a.

Galperin, Yu. I., V. A. Gladyshev, N. V. Jorjio, R. A. Kovrazhkin, Yu. V. Lissakov, V. D. Maslov, L. M. Nikolaenko, R. Z. Sagdeev, O. A. Molchanov, M. M. Mogilevsky, L. S. Alperovich, M. B. Gokhberg, E. A. Ivanov, O. A. Pokhotelov, C. Beghin, J. J. Berthelier, J.-M. Bosqued, and H. Reme, Alfven wave excited in the middle-latitude magnetosphere by a large-scale acoustic wave propagating in lower ionosphere, $I z v$. $A N$ SSSR, Phys. Earth, No. 11, 88-98, 1985b.

Galperin, Yu. I., V. A. Gladyshev, N. V. Jorjio, V. I. Larkina, and M. M. Mogilevsky, Energetic particle precipitation from the magnetosphere above the epicenter of approaching earthquake, Cosmic Res., 30, 89-106, 1992 (in Russian).

Geller, R. J., D. D. Jackson, Ya. Y. Kagan, and F. Mulargia, Earthquakes cannot be predicted, Science, 275, 1616-1617, 1997.

Ginzburg, E. A., A. B. Malyshev, I. P. Proshkina, and V. P. Pustovetov, Correlation of strong earthquakes with variations of particle flux of the radiation belt, Geomagn. Aeron., 34, 60-67, 1994 (in Russian).

Gokhberg, M. B., Strong acoustic wave action, in Active Experiments in Space, ESA Symp. at Alpbach, 24-28, May 1983, edited by ESA, ESASP195, Noordwijk, pp. 99-110, 1983.

Gokhberg, M. B., V. A. Morgunov, T. Yoshino, and T. Tomizawa, Experimental measurements of electromagnetic emissions possibly related to earthquakes in Japan, J. Geophys. Res., 87, No. 9, 7824-7828, 1982.

Gokhberg, M. B., V. A. Pilipenko, and O. A. Pokhotelov, Observations from a satellite of electromagnetic radiation above the region of an earthquake in preparation, Doklady AN SSSR (Reports to the USSR Acad. Sci.), 268, $56-58,1983$.

Gokhberg, M. B., I. L. Gufeld, A. A. Rozhnoy, V. F. Marenko, V. S. Yampolsky, and E. A. Ponomarev, Study of seismic influence on the ionosphere by super long-wave probing of the Earth-ionosphere waveguide, Phys. Earth Planet. Inter., 57, 64-74, 1989.

Gorny, V. I., A. G. Salman, A. A. Tronin, and B. V. Shilin, Outgoing infrared Earth's radiation-an indicator of seismic activity, Doklady An SSSR (Reports to the USSR Acad. Sci.), 301, No. 1, 67-69, 1988.

Greifinger, C. and P. Greifinger, Transient ULF electric and magnetic fields following a lightning discharge, J. Geophys. Res., 81, 2237-2247, 1976.
Gufeld, I. L., G. A. Gusev, R. A. Lyutikov, and M. I. Matveeva, Seismic process as phase instability of the lithosphere, in Atmospheric and Ionospheric Phenomena Associated with Earthquakes, edited by M. Hayakawa, Terra Sci. Pub. Corp., Tokyo, 1998 (in press).

Hayakawa, M., Electromagnetic precursors of earthquakes: Review of recent activities, in Rev. Radio Sci. (1993-1995), edited by W. Ross Stone, pp. 807-818, Oxford Univ. Press, 1997.

Hayakawa, M., I. Tomizawa, K. Ohta, S. Shimakura, Y. K. Fujinawa, K. Takahashi, and T. Yoshino, Direction finding of precursory radio emissions associated with earthquakes: a proposal, Phys. Earth Planet. Inter., 77, 127-135, 1993.

Hayakawa, M., R. Kawate, O. A. Molchanov, and K. Yumoto, Results of ultra-low-frequency magnetic field measurements during the Guam earthquake of 8 August 1993, Geophys. Res. Lett., 23, 241-244, 1996.

Henderson, T. R., V. S. Sonwalkar, R. A. Helliwell, U. S. Inan, and A. C. Fraser-Smith, A search for ELF/VLF emissions induced by earthquakes as observed in the ionosphere by the DE-2 satellite, J. Geophys. Res., 98, 9503-9509, 1993.

Inan, U. S., T. F. Bell, and V. P. Pasko, Reply, Geophys. Res. Lett., 23, 3423-3424, 1996.

Johnston, M. J. S., Review of electrical and magnetic fields accompanying seismic and volcanic activity, Surv. Geophys., 18, 441-475, 1997.

King, C. Ju., Gas chemistry applied to earthquake prediction: An overview, J. Geophys. Res., 91, 12269-12290, 1986.

Larkina, V. I., A. V. Nalivayko, N. I. Gershenzon, M. B. Gokhberg, V. A. Liperovsky, and S. L. Shalimov, Observations of VLF emission related with seismic activity on the Interkosmos-19 satellite, Geomagn. Aeron., 23, 684-687, 1983 (English translation).

Larkina, V. I., V. V. Migulin, O. A. Molchanov, I. P. Kharkov, A. S. Inchin, and V. B. Schvetcova, Some statistical results on very low frequency radiowave emissions in the upper ionosphere over earthquake zones, Phys. Earth Planet. Inter, 57, 100-109, 1989.

Martysh, E. and V. Sidorenko, Upward discharge above seismo-active regions, paper presented at the International Workshop on Seismo-Electromagnetics, Tokyo, March, 1997.

Merzer, M., Close-fit modelling of high ULF magnetic fields preceding Loma Prieta earthquake, 1992 Regional Symposium on Electromagnetic Compatibility, Tel-Aviv, Israel, November, 1992, Program and Papers, 2-1-2, pp. 1-4, 1992.

Molchanov, O. A., Wave and plasma phenomena inside the ionosphere and magnetosphere associated with earthquakes, Rev. Radio Sci., Chap. 28, 591-600, 1993.

Molchanov, O. A., Y. A. Kopytenko, P. V. Voronov, E. A. Kopytenko, T. Matiashvili, A. C. Fraser-Smith, and A. Bernardi, Results of ULF magnetic field measurements near the epicenters of the Spitak $(M s=6.9)$ and Loma-Prieta $(M s=7.1)$ earthquakes: Comparative analysis, Geophys. Res. Lett., 19, 1495-1498, 1992.

Molchanov, O. A., O. A. Mazhaeva, A. N. Goliavin, and M. Hayakawa, Observation by the Intercosmos-24 satellite of ELF-VLF electromagnetic emissions associated with earthquakes, Ann. Geophys., 11, 431440, 1993.

Molchanov, O. A., M. Hayakawa, and V. A. Rafalsky, Penetration characteristics of electromagnetic emissions from an underground seismic source into the atmosphere, ionosphere, and magnetosphere, J. Geophys. Res., 100, A2, 1691-1712, 1995.

Molchanov, O. A., M. Hayakawa, T. Ondoh, and E. Kawai, Precursory effects in the subionospheric VLF signals for the Kobe earthquake, Phys Earth Planet. Inter. 105, 239-248, 1998.

Parrot, M., Statistical study of ELF/VLF emissions recorded by a lowaltitude satellite during seismic events, J. Geophys. Res., 99, 23339 23345, 1994.

Pierce, E. T., Atmospheric electricity and earthquake prediction, Geophys. Res. Lett., 3, 185-188, 1976.

Pokhotelov, O. A., V. A. Pilipenko, E. N. Fedorov, L. Stenflo, and P. K. Shukla, Induced electromagnetic turbulence in the ionosphere and the magnetosphere, Phys. Scr, 50, 600-605, 1994.

Pulinets, S. A., Conjugate seismo-ionospheric variations before strong earthquakes, in Atmospheric and Ionospheric Phenomena Associated with Earthquakes, edited by M. Hayakawa, Terra Sci. Pub. Corp., Tokyo, 1998 (in press).

Qiang Zuji, Thermal infrared anomaly-precursor of impending earthquakes, Chin. Sci. Bull., 36, No. 4, 319-324, 1991.

Rikitake, T., Earthquake prediction: an empirical approach, Tectonophysics, 148, 195-288, 1988.

Roger, C. J., N. R. Thomson, and R. L. Dowden, A search for ELF/VLF 
activity associated with earthquakes using ISIS satellite data, J. Geophys. Res., 101, A6, 13369-13378, 1996.

Roussel-Dupre, R. A., A. V. Gurevich, T. Tunnell, and G. M. Milikh, Kinetic theory of runaway air breakdown, Phys. Rev., 49, No. 3, 2257-2271, 1994

Rowland, H. L., R. F. Fernsler, J. D. Huba, and P. A. Bernhardt, Lightning driven EMP in upper atmosphere, Geophys. Res. Lett., 22, 361-364, 1995.

Sentman, D. D. and E. M. Wescott, Observations of upper atmospheric optical flashes recorded from an aircraft, Geophys. Res. Lett., 20, 2857 2860, 1993.
Sukhorukov, A. I., E. A. Rudenchik, and P. Stubbe, Simulation of the strong lightning pulse penetration into the lower ionosphere, Geophys. Res. Lett., 23, 2911-2914, 1996.

Surkov, V. V., Excitation of low-frequency geomagnetic oscillation upon the propagation of a vertical acoustic wave in the $E$-layer of the ionosphere, Geomagn. Aeron., 32, 332-336, 1992 (English translation).

Winckler, J. R., Further observations of cloud-ionosphere electrical discharges above thunderstorms, J. Geophys. Res., 100, D7, 14335-14345, 1995. 